\title{
Establishing a New Protein X-ray Crystallography Research Group at a PUI
}

Krystle J. McLaughlin, Department of Chemistry, Vassar College

For new principal investigators, setting up a lab and assembling a research group are major goals for the first year. Particular challenges exist when accomplishing this important task at a primarily undergraduate institution (PUI), such as a small liberal arts college, where the resources of a large research institution are not usually available. In this talk, I will discuss in detail the setup from scratch of a new protein crystallography research group at a small liberal arts college. This will include a discussion of anticipated costs, expected timeline, and potential roadblocks. I will also discuss approaches to establishing a productive research group, while examining the challenges unique to running a lab with only undergraduate researchers. 